\title{
COMPARATIVE STUDY BETWEEN HERNIOPLASTY AND HERNIORRHAPHY AT ESI PGIMSR, BASAIDARAPUR, NEW DELHI
}

\section{Surgery}

Dr. Rakesh Kumar M.B.B.S, M.S. (Gen. Surgery), Senior Resident, Department of Surgery, ESI, PGIMSR, Thakur* Basaidarapur, New Delhi. *Corresponding Author

Dr. Tanweer Karim Associate Professor, Department of Surgery, ESI, PGIMSR, Basaidarapur, New Delhi.

\begin{tabular}{ll}
\hline Dr. Debarshi Jana & $\begin{array}{l}\text { Young Scientist (DST), Institute of Post-Graduate Medical Education and Research, } \\
\text { A.J.C. Bose Road, Kolkata, West Bengal, India-700020. }\end{array}$ \\
\hline
\end{tabular}

\section{ABSTRACT}

Inguinal hernia is a very common problem. Surgical repair is the current approach. Local anaesthesia is a suitable and economic option for open repairs, and should be popularized in day-care setting. Numerous repair methods have been described to date. Mesh repairs are superior to "nonmesh" tissue-suture repairs, but in some socio economically backward areas and in rural setups still non-mesh tissue repair is being performed. This study is done to compare and demonstrate the efficacy, acceptability, outcomes and recurrences of herniorrhaphy (Bassinis's repair) and hernioplasty (Lichtenstein's tension free repair). This retrospective comparative study of herniorrhaphy and hernioplasties was conducted in 62 patients who were admitted and treated in surgical setting from August 2014 to July 2015. Patients were evaluated thoroughly with physical examination, routine investigations, and ultrasonography. Patients were selected either for herniorrhaphy or hernioplasty depending on the age of presentation, tone of the abdominal muscles and size of the defect. All the operated patients were followed up for a period of one year. In the study paediatric hernias subjected to herniotomies were excluded. This study showed that majority of the patients who were subjected to herniorrhaphy were patients below the age of 50 years with good abdominal tone and majority of people above 60 years with weak abdominal tone i.e. those presenting with direct hernias, were subjected for hernioplasty and neither of the patient had any recurrence or other complications. Out of the 38 patients who underwent hernioplasty 3 patients had infection and mesh extrusion and 7 had inguinodynia. Out of the 24 patients who underwent herniorrhaphy 4 had scrotal swelling and about 9 of the patients had increased post-operative pain compared to patients who underwent hernioplasties. But no cases of wound infections were reported in Bassini's repair. This study shows that, pre operatively if the patient is assessed properly and then subjected to herniorrhaphy or hernioplasties the rate of recurrence is minimal to nil and more economical. In our study there were no hernia recurrences noticed.

\section{KEYWORDS}

Herniorrhaphy, hernioplasty, abdominal muscle tone.

\section{INTRODUCTION}

A hernia is defined as an area of weakness or complete disruption of the fibromuscular tissues of the body wall. Structures arising from the cavity contained by the body wall can pass through, or herniate, through such a defect. While the definition is straightforward, the terminology is often misrepresented. It should be clear that hernia refers to the actual anatomic weakness or defect, and hernia contents describe those structures that pass through the defect.

Hernias are among the oldest known actions of humankind, and surgical repair of the inguinal hernia is the most common general surgery procedure performed today.

Today, laparoscopic techniques have been validated as safe and effective in the treatment of groin hernias and have become commonplace.

\section{MATERIALS AND METHODS}

This comparative study of herniorrhaphy and hernioplasties was conducted in 62 patients who were admitted and treated in surgical setting from August 2014 to July 2015 at ESI, PGIMSR, Basaidarapur, New Delhi. Patients were evaluated thoroughly with physical examination, routine investigations, and ultrasonography. Patients were selected either for herniorrhaphy or hernioplasty depending on the age of presentation, tone of the abdominal muscles and size of the defect.

All the patients were explained about the procedures preoperatively and consent was taken for the surgery. All the operated patients were followed up for a period of one year.

In the study paediatric hernias subjected to herniotomies were excluded from the study.

\section{RESULTS}

This study showed that majority of the patients who were subjected to herniorrhaphy were patients below the age of 50 years with good abdominal tone and majority of people above 60 years with weak abdominal tone were subjected for hernioplasty. The patients were followed up for 1 year, neither of the patients had any recurrence or other complications.
Even though the number of patients who underwent herniorrhaphy is almost equal to hernioplasties in the study the procedure was performed after carefully assessing the tone of abdominal muscles.

Some of the complications encountered were, post-operative pain was comparatively more in case of patients who underwent herniorrhaphy. But, mesh related infections were encountered in patients who underwent hernioplasty.

Table-1 : Number And Age Of Patients Who Underwent Hernioplasty

\begin{tabular}{|l|l|}
\hline Age Of The Patients & Number Of Hernioplasties \\
\hline$<20$ years & 0 \\
\hline $21-30$ years & 4 \\
\hline $31-40$ years & 3 \\
\hline $41-50$ years & 9 \\
\hline $51-60$ years & 11 \\
\hline$>60$ years & 11 \\
\hline Total & 38 \\
\hline
\end{tabular}

Out of the 38 patients who underwent hernioplasty 3 patients had infection and mesh extrusion and 7 had inguinodynia.

Table-2 : Number And Age Of Patients Who Underwent Herniorrhaphy

\begin{tabular}{|l|l|}
\hline Age Of The Patients & Number Of Herniorrhaphy \\
\hline$<20$ years & 0 \\
\hline $21-30$ years & 5 \\
\hline $31-40$ years & 2 \\
\hline $41-50$ years & 5 \\
\hline $51-60$ years & 5 \\
\hline$>60$ years & 7 \\
\hline Total & 24 \\
\hline
\end{tabular}

Out of the 7 patients aged above 60 years 3 underwent Kuntz repair.

Out of the 24 patients who underwent herniorrhaphy 4 had scrotal swelling and about 9 of the patients had increased post-operative pain compared to patients who underwent hernioplasties.

\section{DISCUSSION}

In the present era of minimally invasive surgeries like laparoscopic 
mesh repair and other recent advance in the field of hernia repair, still in some of the peripheral areas, in rural settings and in economically backward areas still open non mesh hernia repair is the most commonly done procedure and if the patients are selected appropriately after assessing the abdominal muscle tone, the recurrence rate is very minimal and also economical with minimal complications.

\section{Surgical Operations For Inguinal Hemia Are Listed Below:}

The Lichtenstein Tension - free Repair

First reported in 1986 this simple operation consists of suturing a patch of polypropylene mesh to the inguinal ligament below, conjoint tendon above and the pubic tubercle and rectus sheath medially. The mesh is split laterally to accommodate the cord; the 'tails' are crossed over and sutured to each other lateral to the cord. A transverse crease incision and not oblique incision is used. Direct sacs are inverted; indirect sacs are dissected upto the neck but are not ligated. They are also simply inverted or excised. Mesh is anchored with a loose continuous suture.

Unlike surgeons who had reserved prosthetic mesh for "difficult" cases Lichtenstein recommends this procedure for all groin hernias. The reported recurrence rate is 0.2 percent in five different centres.

Some of the complications associated with mesh repair are

- Infection

- Mesh extrusion

- Foreign body reaction

- Mesh inguinodynia causing hyperaesthesia and pain along the distribution of ilioinguinal or iliohypogastric nerves.

- Mesh erosion into bladder, bowel or vessels can occur occasionally

Complications of herniorrhaphy

- Haemorrhage

- Haematoma, seroma

- Infection-1-2\%

- Haematocele

- Post-herniorrhaphy hydrocele, lymphocele

- Hyperaesthesia over the medial side of inguinal canal due to injury to iliohypogastric nerve neuralgia $(15 \%)$

- Recurrence-10-15\%

- Osteitis pubis

- Injury to urinary bladder/bowel

- Testicular atrophy, penile oedema rarely can occur

\section{Gilbert's Suture less Repair}

This is similar to Lichtenstein's repair but the mesh is inserted by a suture less technique.

\section{Stoppa's Giant Prosthetic Reinforcement Of The Visceral Sac- GPRVS}

The essential feature of GPRVS is replacement of the transversalis fascia in the groin and lower abdomen by a large prosthesis that extends far beyond the myopectineal orifice. The prosthesis envelopes the visceral sac, held in place by intra-abdominal pressure (Pascal's Law) and later by connective tissue ingrowth. In theory recurrences after GPRVS are inconceivable; never the less they do occur because of technical errors.

\section{Laparoscopic Hernia Repairs}

\section{Transabdominal Preperitoneal Repair (TAPP):}

After entering the peritoneal cavity by laparoscopy the preperitoneal space is entered, sac dissected free and reduced. Mesh is used to cover the defect by anchoring it to the Cooper's ligament and conjoint tendon. This is the most commonly performed laparoscopic repair

\section{Intraperitoneal Onlay Mesh Repair (IPOM):}

This has largely been abandoned because of complications related to possible mesh erosion into bowel.

\section{Totally Extra-Peritoneal Repair (TEP):}

The mesh is inserted directly into the preperitoneal space. However, a preliminary diagnostic laparoscopy is done.

Scan of current literature shows conflicting reports about the efficacy of TAPP vs TEP. Both the procedures are extensively used all over the world.

The advantages of laparoscopic repair are: -

- Reduced post operative pain and disability
- Inguinal and femoral hernias can be inspected bi-laterally

- Bilateral hernias can be repaired in one sitting

- An unsuspected contralateral hernia can be detected and repaired in the same sitting.

- It avoids the operative site in recurrent hernias

The disadvantages are: -

- Violation of peritoneal cavity

- Need for general anaesthesia

- Cost

- Need for expertise

In this comparative study of herniorrhaphy and hernioplasties patients were evaluated thoroughly with physical examination, routine investigations, and ultrasonography. Patients were selected either for herniorrhaphy or hernioplasty depending on the age of presentation, tone of the abdominal muscles and size of the defect.

All the patients were explained about the procedures preoperatively and consent was taken for the surgery.

Patients above the age of 50 years with poor abdominal tone were selected for hernioplasties and some of them were selected for herniorrhaphies with orchidectomy (Kuntz procedure) and some for Hamilton Bailey's procedure where in the testis are retained with excision of the cord and complete closure of the deep ring is done. And those patients below 50 years who had good abdominal tone were selected for herniorrhaphies, and if intraoperatively increased tension was noted along the repair site Tanner's sliding incision (To reduce the tension in the repair area, relaxing incision is placed over the lower rectus sheath so that conjoined tendon is allowed to slide downward) was given and in cases of strangulated hernias, herniorrhaphy was done and not hernioplasty in view of contamination and mesh infection.

In this study all the patients who underwent herniorrhaphies and hernioplasties were followed up for a period of 1 year during which no recurrences have been reported. and some of the post operative complications which were encountered for herniorrhaphies were scrotal swelling which was treated with scrotal support and other being increased post operative pain compared to hernioplasties which was treated with analgesics.

Inguinodynia (It is chronic inguinal pain seen in post-hernia surgery patients) was seen which was managed conservatively with analgesics.

\section{CONCLUSION:}

This comparative study shows that after appropriate investigations and clinical examination, patients can be selected either for herniorrhaphy or hernioplasty.In young people with good abdominal tone and with indirect hernia still herniorrhaphy can be considered as an appropriate procedure. It is also cost effective and the risks of mesh repair can be avoided. Whereas in old people or in patients with weak abdominal tone i.e. those with direct hernia, hernioplasty would be the surgery of choice.

\section{REFERENCES}

1. Bassini E. Nuovo metodo perla cura radicale dell'ernia inguinale. Atti Congr Assoc Med Ital. $1887 ; 2: 179$

2. Gilbert AI. An anatomic and functional classification for the diagnosis and treatment of inguinal hernia. The American journal of surgery. 1989 Mar 1;157(3):331-3

3. Lichtenstein IL, Shulman AG, Amid PK, Montllor MM. The tension-free hernioplasty. The American Journal of Surgery. 1989 Feb 1;157(2):188-93.

4. Rutkow IM, Robbins AW. Demographic, classificatory, and socioeconomic aspects of Rernia repair in the United States. Surgical Clinics of North America. 1993 Jun 30;73(3):413-26.

5. Stloppa R. Classification of hernia. In :Chevrcl JP editor. Hernias and Surgery of the Abdominal wall, 1st ed. Berlin : Springer- Vcrlag. 1998:175-84

6. Stoppa RE. The preperitoneal approach and prosthetic repair of groin hernia. In Nyhu LM. Condon RE. editors. Hernia. 4th ed. Philadelphia :Lippincott. 1995.P 188-204. Topal B. Hourlay P. Totally preperitoneal endoscopic ingui-nal hernia repair. Br J Surg 1997;84(1):6I-3

8. Trans abdominal or totally extraperitoneal laparoscopic hernia repair? Surg Laparosc Endosc 1998;8<4):264-8 